\title{
Local Dynamics and Global Stability of Certain Second-Order Rational Difference Equation with Quadratic Terms
}

\author{
S. Jašarević Hrustić, ${ }^{1}$ M. R. S. Kulenović, ${ }^{2}$ and M. Nurkanović ${ }^{1}$ \\ ${ }^{1}$ Department of Mathematics, University of Tuzla, 75000 Tuzla, Bosnia and Herzegovina \\ ${ }^{2}$ Department of Mathematics, University of Rhode Island, Kingston, RI 02881-0816, USA
}

Correspondence should be addressed to M. R. S. Kulenović; mkulenovic@mail.uri.edu

Received 5 August 2015; Accepted 1 December 2015

Academic Editor: Garyfalos Papashinopoulos

Copyright (C) 2016 S. Jašarević Hrustić et al. This is an open access article distributed under the Creative Commons Attribution License, which permits unrestricted use, distribution, and reproduction in any medium, provided the original work is properly cited.

We present a complete local dynamics and investigate the global dynamics of the following second-order difference equation: $x_{n+1}=\left(A x_{n}^{2}+E x_{n-1}+F\right) /\left(a x_{n}^{2}+e x_{n-1}+f\right), n=0,1,2, \ldots$, where the parameters $A, E, F, a, e$, and $f$ are nonnegative numbers with condition $A+E+F>0, a+e+f>0$, and the initial conditions $x_{-1}, x_{0}$ are arbitrary nonnegative numbers such that $a x_{n}^{2}+e x_{n-1}+f>0, n=0,1,2, \ldots$

\section{Introduction and Preliminaries}

In this paper we investigate the local and global dynamics of the following difference equation:

$$
x_{n+1}=\frac{A x_{n}^{2}+E x_{n-1}+F}{a x_{n}^{2}+e x_{n-1}+f}, \quad n=0,1,2, \ldots,
$$

where the parameters $A, E, F, a, e$, and $f$ are nonnegative numbers with condition $A+E+F>0, a+e+f>0$, and the initial conditions $x_{-1}, x_{0}$ are arbitrary nonnegative numbers such that $a x_{n}^{2}+e x_{n-1}+f>0, n=0,1,2, \ldots$ The change of variable $x_{n}=1 / u_{n}$ transforms (1) to the following difference equation:

$$
u_{n+1}=\frac{f u_{n}^{2} u_{n-1}+e u_{n}^{2}+a u_{n-1}}{F u_{n}^{2} u_{n-1}+E u_{n}^{2}+A u_{n-1}}, \quad n=0,1, \ldots
$$

where we assume that $F+E+A>0$ and that the nonnegative initial conditions $u_{-1}, u_{0}$ are such that $F u_{n}^{2} u_{n-1}+E u_{n}^{2}+A u_{n-1}>$ 0 for all $n \geq 0$. Thus the results of this paper extend to (2).
Equation (1) is the special case of a general second-order quadratic fractional equation of the form

$$
\begin{array}{r}
x_{n+1}=\frac{A x_{n}^{2}+B x_{n} x_{n-1}+C x_{n-1}^{2}+D x_{n}+E x_{n-1}+F}{a x_{n}^{2}+b x_{n} x_{n-1}+c x_{n-1}^{2}+d x_{n}+e x_{n-1}+f}, \\
n=0,1,2, \ldots,
\end{array}
$$

with nonnegative parameters and initial conditions such that $A+B+C>0, a+b+c+d+e+f>0$, and $a x_{n}^{2}+b x_{n} x_{n-1}+$ $c x_{n-1}^{2}+d x_{n}+e x_{n-1}+f>0, n=0,1, \ldots$

Some special cases of (1) were investigated in [1], where special case $A=a=0$, known as Riccati's equation, was thoroughly investigated and its global dynamics was completely described. In fact, this is one of very few nonlinear difference equations whose solution can be explicitly found. As it was shown in [1] such equation can exhibit the whole range of different global behaviors such as global asymptotic stability of the equilibrium, global periodicity (i.e., all solutions are periodic with the same period), and chaos. To avoid this case we assume that $A+a>0$. Furthermore, the special case $E=$ $e=0$ is the first-order difference equation whose dynamics is well known and follows from Theorem C.3, page 484 in [2]. Consequently, we will assume that $E+e>0$. Finally, the special case $a=e=0$, known as the Henon difference equation [3], was completely solved in [4], where the basins 
of attraction of all equilibrium points and the point at infinity were found and global dynamics was described in full detail. So, in this paper we assume that $a+e>0$.

The first systematic study of global dynamics of a special quadratic fractional case of (3) where $A=C=D=a=$ $c=d=0$ was performed in $[5,6]$. Dynamics of some related quadratic fractional difference equations was considered in the papers [7-17]. In this paper we will perform the local stability analysis of all three equilibrium points of (1) and we will give the necessary and sufficient conditions for the equilibrium to be locally asymptotically stable, a saddle point, a repeller, or a nonhyperbolic equilibrium. The local stability analysis indicates that some possible dynamics scenarios for (1) include period doubling bifurcations, as in the case of equation

$$
x_{n+1}=\frac{x_{n-1}}{a x_{n}^{2}+e x_{n-1}+f}, \quad n=0,1,2, \ldots
$$

considered in [18]. Another possible behavior is chaos as in the case of equation

$$
x_{n+1}=\frac{A x_{n}^{2}+F}{x_{n-1}}, \quad n=0,1,2, \ldots,
$$

considered in [19]. Our local stability analysis will exclude the possibility of Neimark-Sacker bifurcation.

The paper is organized as follows. The rest of this section contains some global attractivity results which will be used in Section 4 to obtain global asymptotic stability results for some special cases of (1). Section 2 gives local stability analysis of all three equilibrium points when $F>0$ and Section 3 gives local stability analysis of all equilibrium points when $F=$ 0 . Finally, Section 4 gives some global attractivity results in some special cases. The global attractivity and global stability results that will be used in Section 4 are Theorems 1.4 .5 and 1.4.7 from [1] and four results that follow.

The global attractivity results obtained specifically for complicated cases of (3), when many terms in numerator and denominator are present, are the following theorems [20].

Theorem 1. Assume that (3) has the unique equilibrium $\bar{x}$. If the condition

$$
\begin{aligned}
& \frac{(|A-a \bar{x}|+|B-b \bar{x}|+|C-c \bar{x}|)(U+\bar{x})+|D-d \bar{x}|+|E-e \bar{x}|}{(a+b+c) L^{2}+(d+e) L+f} \\
& \quad<1
\end{aligned}
$$

holds, where $L$ and $U$ are lower and upper bounds of all solutions of (3), then $\bar{x}$ is globally asymptotically stable.

Theorem 2. Assume that (3) has the unique equilibrium $\bar{x}$. If the condition

$$
\begin{gathered}
(|A-a \bar{x}|+|B-b \bar{x}|+|C-c \bar{x}|)(M+\bar{x})+|D-d \bar{x}| \\
+|E-e \bar{x}|<(a+b+c) m^{2}+(d+e) m+f
\end{gathered}
$$

holds, where $m=\min \left\{\bar{x}, x_{-1}, x_{0}\right\}$ and $M=\max \left\{\bar{x}, x_{-1}, x_{0}\right\}$ are lower and upper bounds of specific solution of (3), then $\bar{x}$ is globally asymptotically stable on the interval $[m, M]$.
In the case of (1) Theorems 1 and 2 give the following special results.

Corollary 3. If the condition

$$
\frac{|A-a \bar{x}|(U+\bar{x})+|E-e \bar{x}|}{a L^{2}+e L+f}<1
$$

holds, where $L$ and $U$ are lower and upper bounds of all solutions of (1), then $\bar{x}$ is globally asymptotically stable.

Corollary 4. If the condition

$$
|A-a \bar{x}|(M+\bar{x})+|E-e \bar{x}|<a m^{2}+e m+f
$$

holds, where $m=\min \left\{\bar{x}, x_{-1}, x_{0}\right\}$ and $M=\max \left\{\bar{x}, x_{-1}, x_{0}\right\}$ are lower and upper bounds of specific solution of (1), then $\bar{x}$ is globally asymptotically stable on the interval $[m, M]$.

\section{Case $F>0$}

This section gives complete local stability analysis for all equilibrium points (up to three) of (1) when $F>0$.

2.1. Equilibrium Points. Equilibrium points of (1) are the positive solutions of the equation

$$
\bar{x}=\frac{A \bar{x}^{2}+E \bar{x}+F}{a \bar{x}^{2}+e \bar{x}+f}
$$

or equivalently

$$
a \bar{x}^{3}+(e-A) \bar{x}^{2}+(f-E) \bar{x}-F=0 .
$$

Theorem 5. (a) Equation (1) has a unique equilibrium point if one of the following conditions holds:

(1) $(A-e)^{2}>3 a(f-E)$ and $[A<e$ or $(A>e, f \leq E)]$;

(2) $(A-e)^{2}>3 a(f-E), A>e, f>E$, and

$$
\begin{aligned}
& (f-E)^{2}\left[4 a(f-E)-(e-A)^{2}\right] \\
& \quad+F(A-e)\left[4(A-e)^{2}-18 a(f-E)\right] \\
& \quad+27 a^{2} F^{2}>0 ;
\end{aligned}
$$

(3) $(A-e)^{2} \leq 3 a(f-E)$;

(4) $A=e$.

See Figures 1-3.

(b) Equation (1) has two equilibrium points if $(A-e)^{2}>$ $3 a(f-E), A>e, f>E$, and

$$
\begin{aligned}
F= & -\frac{2}{27 a^{2}}(A-e)^{2} \\
& \cdot\left(A-e-\sqrt{(A-e)^{2}+3 a(E-f)}\right) \\
& -\frac{1}{9 a}(f-E) \\
& \cdot\left[3(e-A)+2 \sqrt{(A-e)^{2}+3 a(E-f)}\right]
\end{aligned}
$$




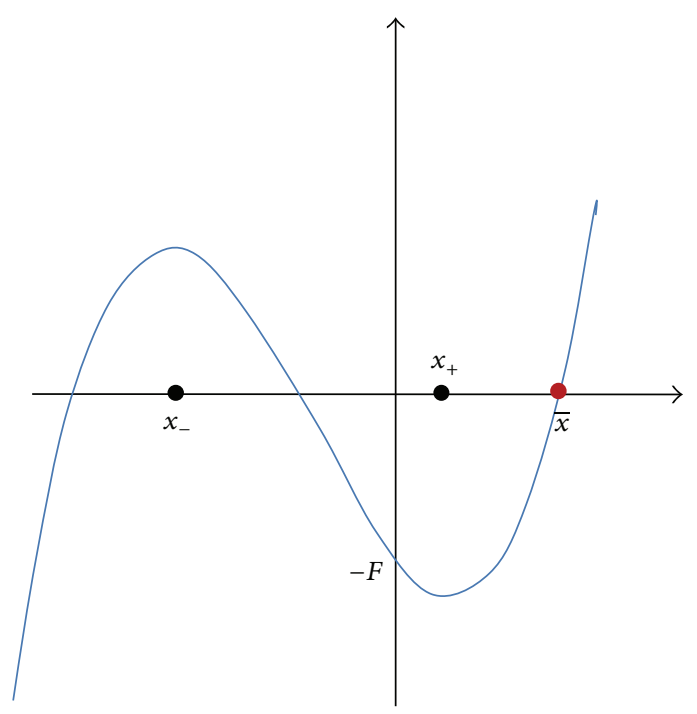

(a)

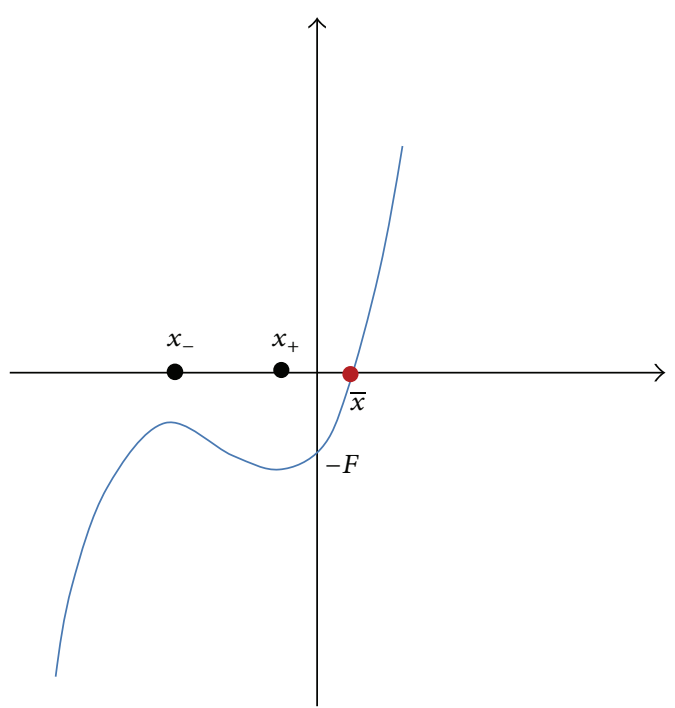

(b)

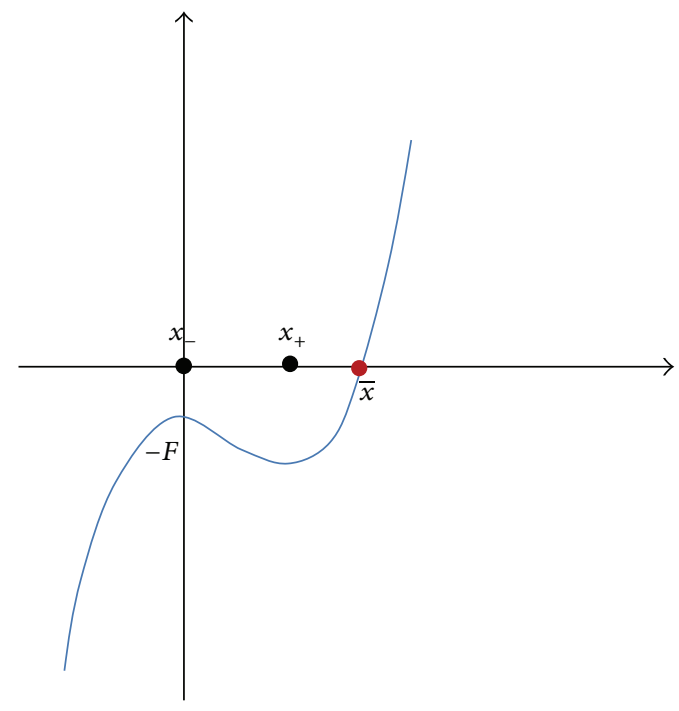

(c)

FIGURE 1

or

$$
\begin{aligned}
F= & -\frac{2}{27 a^{2}}(A-e)^{2} \\
& \cdot\left(A-e+\sqrt{(A-e)^{2}+3 a(E-f)}\right) \\
& +\frac{1}{9 a}(f-E) \\
& \cdot\left[3(A-e)+2 \sqrt{(A-e)^{2}+3 a(E-f)}\right] .
\end{aligned}
$$

(c) Equation (1) has three equilibrium points if $(A-e)^{2}>$ $3 a(f-E), A>e, f>E$, and

$$
\begin{aligned}
& (f-E)^{2}\left[4 a(f-E)-(e-A)^{2}\right] \\
& \quad+F(A-e)\left[4(A-e)^{2}-18 a(f-E)\right] \\
& \quad+27 a^{2} F^{2}<0 .
\end{aligned}
$$

See Figure 4(c).

Proof. Denote by

$$
G(x)=a x^{3}+(e-A) x^{2}+(f-E) x-F .
$$

It is easy to see that $G(-\infty)=-\infty, G(\infty)=\infty, G(0)=-F$, and

$$
G^{\prime}(x)=3 a x^{2}+2(e-A) x+f-E .
$$




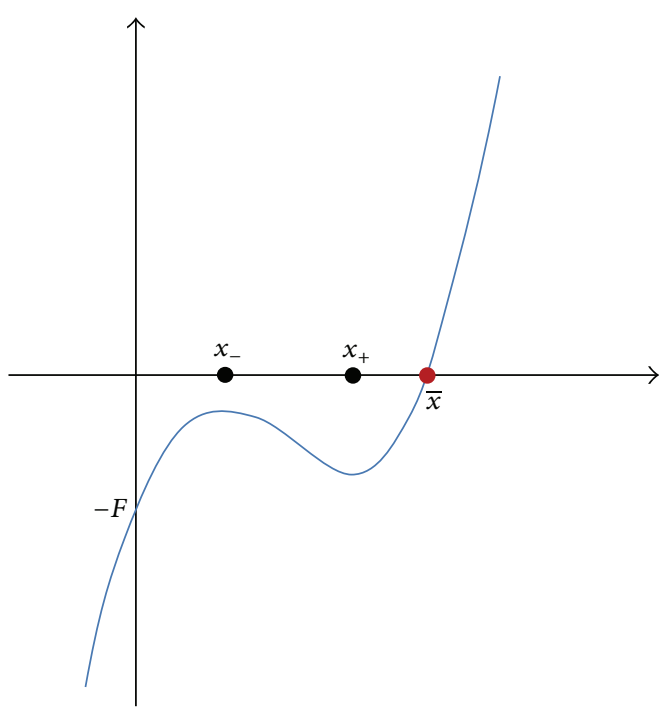

(a)

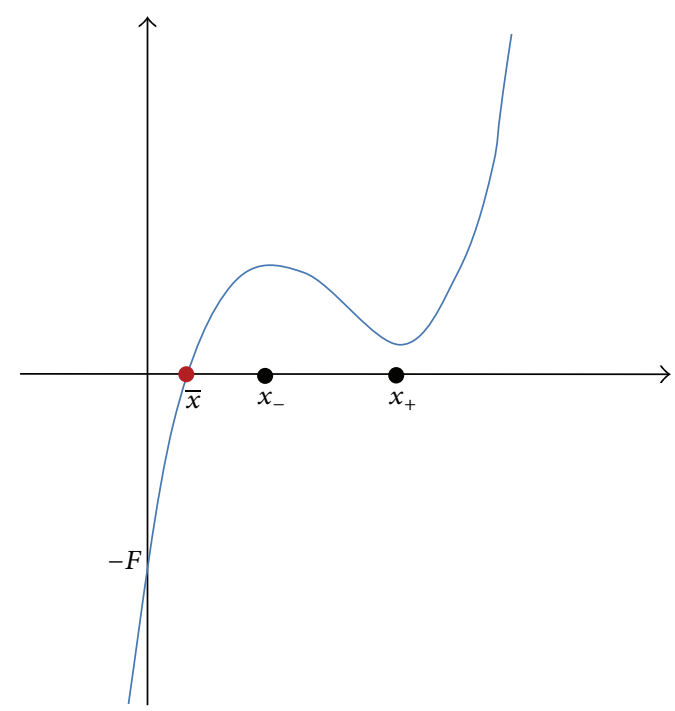

(b)

Figure 2

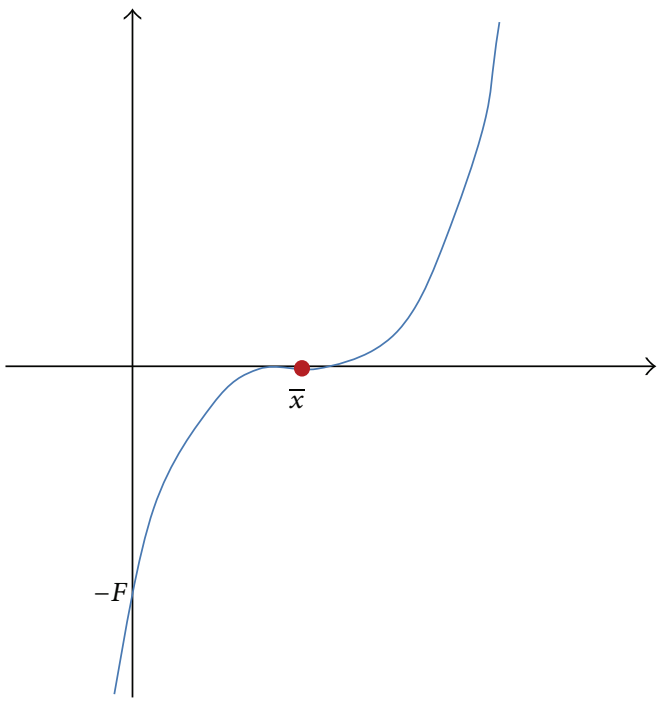

(a)

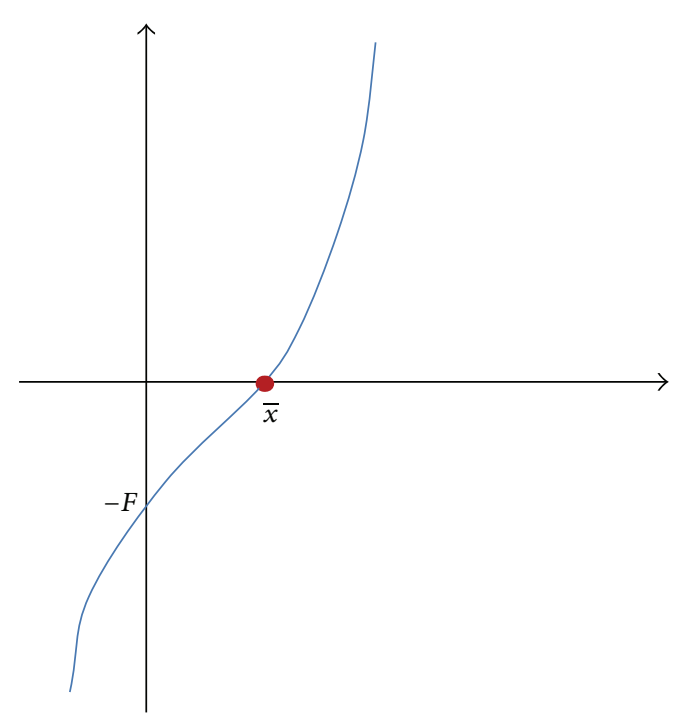

(b)

Figure 3

The critical points of $G$ satisfy

$$
G^{\prime}(x)=0 \Longleftrightarrow 3 a x^{2}+2(e-A) x+f-E=0
$$

and are given as

$$
\begin{aligned}
& x_{ \pm}=\frac{A-e \pm \sqrt{(e-A)^{2}-3 a(f-E)}}{3 a}, \\
& \text { if }(A-e)^{2}>3 a(f-E) .
\end{aligned}
$$

Notice that $G_{\max }=G\left(x_{-}\right), G_{\min }=G\left(x_{+}\right)$, and $x_{-}<x_{+}$.

(a)

(1) Let $(A-e)^{2}>3 a(f-E)$. (i) If $A<e, f \leq E$, then $x_{-}<0<x_{+}$which implies that function $G(x)$ intersects the positive part of $x$-axis in one point since $G(0)=-F$.

(ii) If $A<e, f>E$, then $x_{-}<0, x_{+}<0$ which implies that function $G(x)$ intersects the positive part of $x$-axis in one point since $G(0)=$ $-F$.

(iii) If $A>e, f=E$, then $x_{+}>x_{-}=0$. Using the fact that $G\left(x_{-}\right)=G(0)=-F$ we conclude that (1) has the unique equilibrium point.

(iv) If $A>e, f<E$, then $x_{+}>0>x_{-}$, since $G(0)=$ $-F(1)$ has the unique positive equilibrium point.

(2) Let $(A-e)^{2}>3 a(f-E)$.

If $A>e, f>E$, then $x_{-}>0, x_{+}>0$, and there exists the unique equilibrium point $\bar{x}$ if $G\left(x_{-}\right)<0$ 


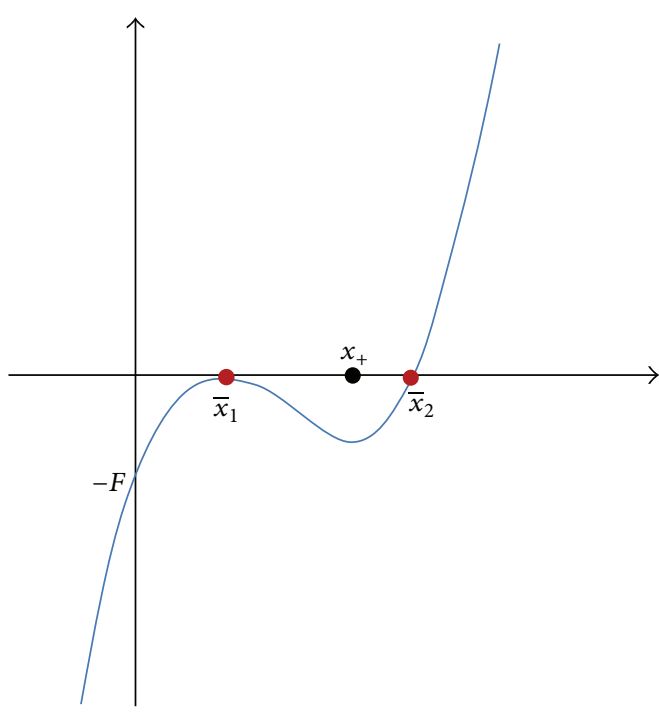

(a)

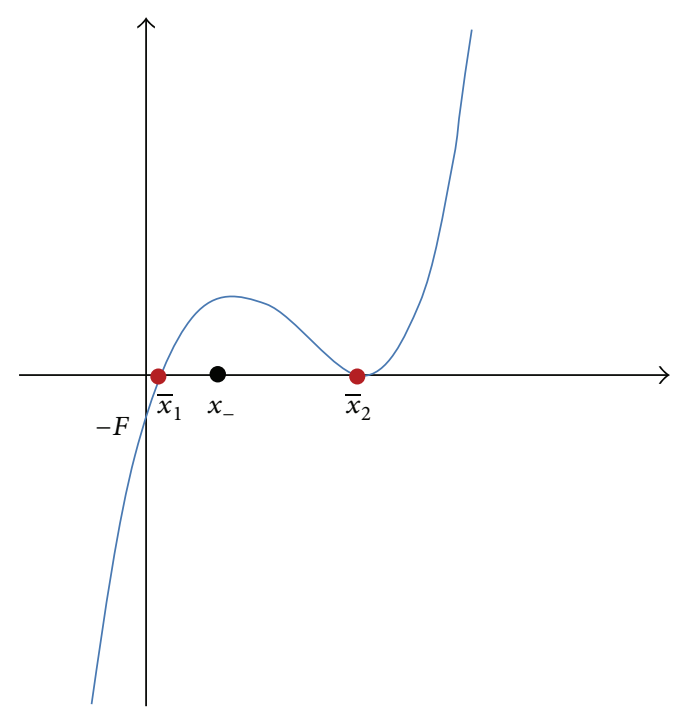

(b)

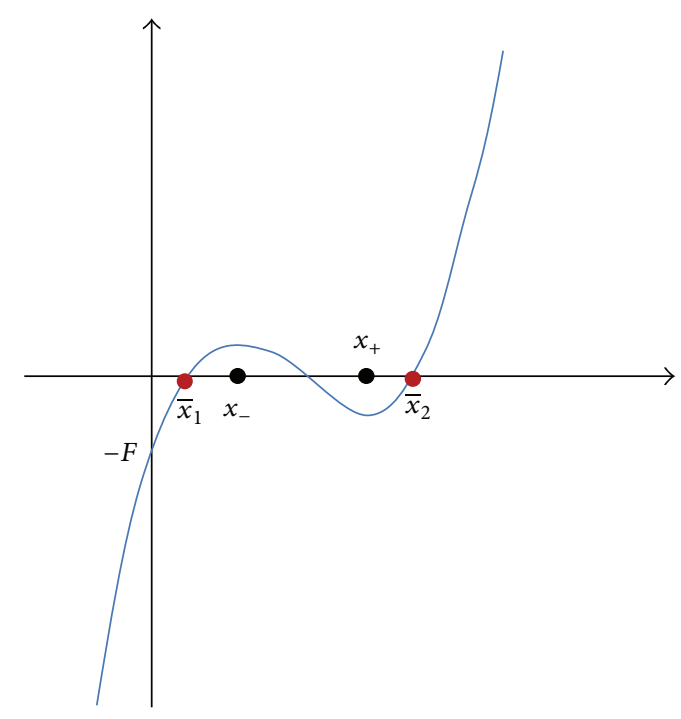

(c)

Figure 4

or $G\left(x_{+}\right)>0$; that is, $G\left(x_{-}\right) G\left(x_{+}\right)>0$ which is equivalent to

$$
\begin{aligned}
& (f-E)^{2}\left[4 a(f-E)-(e-A)^{2}\right] \\
& \quad+F(A-e)\left[4(A-e)^{2}-18 a(f-E)\right] \\
& \quad+27 a^{2} F^{2}>0 .
\end{aligned}
$$

(3) (i) If $(A-e)^{2}<3 a(f-E), G^{\prime}(x)>0, \forall x \in(-\infty, \infty)$ which implies that $G(x)$ is increasing function. Since $G(0)=-F<0$ there exists the unique positive equilibrium point.

(ii) If $(A-e)^{2}=3 a(f-E)$ then $x_{ \pm}=(A-e) / 3 a$ and $G^{\prime \prime}\left(x_{ \pm}\right)=0$ which implies that $x_{ \pm}$is an inflection point of $G$. Since $G(0)=-F$, (1) has only one positive equilibrium point.
(4) (i) If $A=e, f \geq E$, then $G^{\prime}(x)=3 a x^{2}+f-E>0$ and $G(x)$ is increasing function. Since $G(0)=$ $-F$, there exists only one positive equilibrium point.

(ii) If $A=e, f<E \Rightarrow x_{+}= \pm \sqrt{(E-f) / 3 a}$, and $G\left(x_{+}\right)<0$ which implies that there exists only one positive equilibrium point.

(b) If $(A-e)^{2}>3 a(f-E)$ and $A>e, f>E$, then both stationary points $x_{ \pm}$are positive. If

$$
\begin{aligned}
F= & -\frac{2}{27 a^{2}}(A-e)^{2} \\
& \cdot\left(A-e-\sqrt{(A-e)^{2}+3 a(E-f)}\right) \\
& -\frac{1}{9 a}(f-E) \\
& \cdot\left[3(e-A)+2 \sqrt{(A-e)^{2}+3 a(E-f)}\right]
\end{aligned}
$$


or

$$
\begin{aligned}
F= & -\frac{2}{27 a^{2}}(A-e)^{2} \\
& \cdot\left(A-e+\sqrt{(A-e)^{2}+3 a(E-f)}\right) \\
& +\frac{1}{9 a}(f-E) \\
& \cdot\left[3(A-e)+2 \sqrt{(A-e)^{2}+3 a(E-f)}\right]
\end{aligned}
$$

then $G\left(x_{-}\right)=0$ or $G\left(x_{+}\right)=0$ and (1) has exactly two equilibrium points. In the first case $\bar{x}_{2}>\bar{x}_{1}=x_{-}$. In the second case $\bar{x}_{1}<\bar{x}_{2}=x_{+}$.

(c) If $(A-e)^{2}>3 a(f-E)$ and $A>e, f>E$, then both stationary points $x_{ \pm}$are positive. If $G\left(x_{-}\right)>0$ and $G\left(x_{+}\right)<0$ which is equivalent to

$$
\begin{aligned}
& (f-E)^{2}\left[4 a(f-E)-(e-A)^{2}\right] \\
& \quad+F(A-e)\left[4(A-e)^{2}-18 a(f-E)\right] \\
& \quad+27 a^{2} F^{2}<0,
\end{aligned}
$$

(1) has exactly three equilibrium points.

See Table 1 for existence of the positive equilibrium points of (1) when $F>0$.

2.2. Local Stability Analysis. In this section we present the local stability of equilibrium points of (1) in the case $F>0$.

Set

$$
H(u, v)=\frac{A u^{2}+E v+F}{a u^{2}+e v+f}
$$

If $\bar{x}$ denotes an equilibrium point of (1), then the linearized equation associated with (1) about the equilibrium point $\bar{x}$ is

$$
z_{n+1}=p z_{n}-q z_{n-1}
$$

where

$$
\begin{aligned}
p & =H_{u}^{\prime}(\bar{x}, \bar{x})=\frac{2 A \bar{x}(e \bar{x}+f)-2 a \bar{x}(E \bar{x}+F)}{\left(a \bar{x}^{2}+e \bar{x}+f\right)^{2}}, \\
-q & =H_{v}^{\prime}(\bar{x}, \bar{x})=\frac{E\left(a \bar{x}^{2}+f\right)-e\left(A \bar{x}^{2}+F\right)}{\left(a \bar{x}^{2}+e \bar{x}+f\right)^{2}} .
\end{aligned}
$$

Theorem 6. Assume that $F>0$ and let $M=(A+e)^{2}+a(E+f)$. If any of the conditions

(1) $(A-e)^{2}<3 a(f-E)$,

(2) $(A-e)^{2}=3 a(f-E), e<A$, and $F \neq\left(1 / 27 a^{2}\right)(A-e)^{3}$,

(3) $(A-e)^{2}>3 a(f-E), E>f$,

(4) $(A-e)^{2}>3 a(f-E), E \leq f, e>A$,

(5) $(A-e)^{2}>3 a(f-E), E<f, e<A$, and

$$
\begin{aligned}
(E- & f)^{2}\left(-(e-A)^{2}-4 a(E-f)\right) \\
& +F(A-e)\left(4(A-e)^{2}+18 a(E-f)\right) \\
& +27 a^{2} F^{2}>0
\end{aligned}
$$

is satisfied, then the unique equilibrium point $\bar{x}$ of (1) is

(a) locally asymptotically stable if

$$
\begin{aligned}
2 A^{3}+ & 6 e^{3}+a e(3 E+5 f) \\
& +\left(6 e^{2}+2 A^{2}+8 A e+2 a f\right) \sqrt{M}+10 A^{2} e \\
& +14 A e^{2}+a A(E+3 f)-a^{2} F>0,
\end{aligned}
$$

(b) a nonhyperbolic point if

$$
\begin{aligned}
2 A^{3}+ & 6 e^{3}+a e(3 E+5 f) \\
& +\left(6 e^{2}+2 A^{2}+8 A e+2 a f\right) \sqrt{M}+10 A^{2} e \\
& +14 A e^{2}+a A(E+3 f)-a^{2} F=0,
\end{aligned}
$$

(c) a saddle point if

$$
\begin{aligned}
2 A^{3}+ & 6 e^{3}+a e(3 E+5 f) \\
& +\left(6 e^{2}+2 A^{2}+8 A e+2 a f\right) \sqrt{M}+10 A^{2} e \\
& +14 A e^{2}+a A(E+3 f)-a^{2} F<0 .
\end{aligned}
$$

Proof. By using (26) and (27) we get

$$
\begin{aligned}
p-1-q & =\frac{-3 a \bar{x}^{2}+(2 A-2 e) \bar{x}+E-f}{a \bar{x}^{2}+e \bar{x}+f} \\
& =\frac{-G^{\prime}(\bar{x})}{a \bar{x}^{2}+e \bar{x}+f},
\end{aligned}
$$

$$
p+1+q=\frac{-a \bar{x}^{2}+(2 A+2 e) \bar{x}+E+f}{a \bar{x}^{2}+e \bar{x}+f} .
$$

It is clear that $a \bar{x}^{2}+e \bar{x}+f>0$. If the hypotheses of theorem are satisfied, function $G$ is increasing as it passes through the unique equilibrium point $\bar{x}$. Therefore $G^{\prime}(\bar{x})>0$ which implies that $p-1-q<0$. 
TABLE 1: Existence of the positive equilibrium points of (1) when $F>0$.

\begin{tabular}{|c|c|c|c|}
\hline$(A-e)^{2}>3 a(f-E)$ & $A<e$ & $\bar{x}>x_{+}$ & \multirow{7}{*}{ One equilibrium point } \\
\hline$(A-e)^{2}>3 a(f-E)$ & $A>e, f \leq E$ & $\bar{x}>x_{+}>0$ & \\
\hline$(A-e)^{2}>3 a(f-E)$ & $A>e, f>E$ & $\bar{x}>x_{+},\left(G\left(x_{-}\right)<0\right)$ & \\
\hline & $G\left(x_{-}\right) G\left(x_{+}\right)>0$ & $\bar{x}<x_{-},\left(G\left(x_{-}\right)>0\right)$ & \\
\hline$(A-e)^{2}=3 a(f-E)$ & & $\bar{x}=\frac{A-e}{3 a}$ & \\
\hline \multirow[t]{2}{*}{$(A-e)^{2}<3 a(f-E)$} & & $\bar{x}>0$ & \\
\hline & $A=e$ & $\bar{x}>0$ & \\
\hline$(A-e)^{2}>3 a(f-E)$ & $A>e, f>E, G\left(x_{-}\right)=0$ & $\bar{x}_{1}=x_{-}, \bar{x}_{2}>x_{+}$ & \multirow{2}{*}{ Two equilibrium points } \\
\hline$(A-e)^{2}>3 a(f-E)$ & $A>e, f>E, G\left(x_{+}\right)=0$ & $\bar{x}_{1}<x_{-}, \bar{x}_{2}=x_{+}$ & \\
\hline$(A-e)^{2}>3 a(f-E)$ & $A>e, f>E, G\left(x_{-}\right)>0, G\left(x_{+}\right)<0$ & $\bar{x}_{1}<x_{-}<\bar{x}_{2}<x_{+}<\bar{x}_{3}$ & Three equilibrium points \\
\hline
\end{tabular}

Next

$$
m_{1,2}=\frac{1}{a}\left(A+e \pm \sqrt{(A+e)^{2}+a(E+f)}\right)
$$

are zeros of the quadratic function $-a x^{2}+(2 A+2 e) x+E+f$.

It is clear that $m_{1}<0, m_{2}>0$. Notice that $p+1+q>0$ is equivalent to $\bar{x}<m_{2}$ which is equivalent to $G\left(m_{2}\right)>0$.

One can see that $G\left(m_{2}\right)>0$ if and only if

$$
\begin{aligned}
2 A^{3}+ & 6 e^{3}+a e(3 E+5 f) \\
& +\left(6 e^{2}+2 A^{2}+8 A e+2 a f\right) \sqrt{M}+10 A^{2} e \\
& +14 A e^{2}+a A(E+3 f)-a^{2} F>0 .
\end{aligned}
$$

Similarly $p+1+q=0$ if $\bar{x}=m_{2}$ which is equivalent to $G\left(m_{2}\right)=0$. Finally $p+1+q<0$ if $\bar{x}>m_{2}$ which is equivalent to $G\left(m_{2}\right)<0$. The conclusion follows from Theorem 1.1 in [1].

Theorem 7. If $(A-e)^{2}=3 a(f-E), e<A$, and $F=$ $\left(1 / 27 a^{2}\right)(A-e)^{3}$, then the unique positive equilibrium point $\bar{x}=(A-e) / 3 a$ is nonhyperbolic.

Proof. If we assume that $(A-e)^{2}=3 a(f-E)$, then $x_{ \pm}=$ $(A-e) / 3 a$. If $F=(A-e)^{3} / 27 a^{2}$ then

$$
\begin{aligned}
& G\left(x_{ \pm}\right)=\frac{1}{27 a^{2}}\left(-2 A^{3}+6 A^{2} e+2 e^{3}+9 a e(E-f)\right. \\
& \left.-6 A e^{2}+9 a A(f-E)-27 a^{2} F\right) \\
& =\frac{1}{27 a^{2}}\left(-2(A-e)^{3}+9 a(E-f)(e-A)\right. \\
& \left.-27 a^{2} F\right)=\frac{1}{27 a^{2}}\left((A-e)^{3}-27 a^{2} F\right)=0
\end{aligned}
$$

which means that $\bar{x}=x_{ \pm}$is positive equilibrium point since $e<A$. For the equilibrium point $\bar{x}$ we have that

$$
\begin{aligned}
p-1-q & =\frac{-3 a \bar{x}^{2}+(2 A-2 e) \bar{x}+E-f}{a \bar{x}^{2}+e \bar{x}+f} \\
& =\frac{-G^{\prime}(\bar{x})}{a \bar{x}^{2}+e \bar{x}+f}=0
\end{aligned}
$$

which implies that $\bar{x}$ is nonhyperbolic equilibrium point.
Lemma 8. Let $q$ be the partial derivative given by (27). Then $-1<q<1$.

Proof. Inequality $q<1$ is equivalent to

$$
e\left(A \bar{x}^{2}+F\right)-E\left(a \bar{x}^{2}+f\right)<\left(a \bar{x}^{2}+e \bar{x}+f\right)^{2} .
$$

Using (10) we have that

$$
\begin{aligned}
& e\left(a \bar{x}^{3}+e \bar{x}^{2}+f \bar{x}-E \bar{x}\right)-E\left(a \bar{x}^{2}+f\right) \\
& \quad<\left(a \bar{x}^{2}+e \bar{x}+f\right)^{2} \Longleftrightarrow \\
& -a^{2} \bar{x}^{4}-a e \bar{x}^{3}-(E+2 f) a \bar{x}^{2}-(f+E) e \bar{x}-E f \\
& \quad-f^{2}<0,
\end{aligned}
$$

which is satisfied for all values of parameters.

Inequality $q>-1$ is equivalent to

$$
\begin{aligned}
& q>-1 \Longleftrightarrow \\
& e\left(A \bar{x}^{2}+F\right)-E\left(a \bar{x}^{2}+f\right)>-\left(a \bar{x}^{2}+e \bar{x}+f\right)^{2} \Longleftrightarrow(10) \\
& e\left(a \bar{x}^{3}+e \bar{x}^{2}+f \bar{x}-E \bar{x}\right)-E a \bar{x}^{2}-E f \\
& \quad+\left(a \bar{x}^{2}+e \bar{x}+f\right)^{2}>0 \Longleftrightarrow \\
& e a \bar{x}^{3}+e^{2} \bar{x}^{2}+e f \bar{x} \\
& \quad+\left(a \bar{x}^{2}+e \bar{x}+f\right)\left(a \bar{x}^{2}+e \bar{x}+f-E\right) \Longleftrightarrow \\
& e a \bar{x}^{3}+e^{2} \bar{x}^{2}+e f \bar{x} \\
& \quad+\left(a \bar{x}^{2}+e \bar{x}+f\right)\left(A \bar{x}+E+\frac{F}{\bar{x}}-E\right)>0 \Longleftrightarrow \\
& e a \bar{x}^{3}+e^{2} \bar{x}^{2}+e f \bar{x}+\left(a \bar{x}^{2}+e \bar{x}+f\right)\left(A \bar{x}+\frac{F}{\bar{x}}\right)>0,
\end{aligned}
$$

which is true.

The consequence of Lemma 8 is that $\bar{x}$ cannot be a repeller. 
Theorem 9. (a) If $(A-e)^{2}>3 a(f-E), A>e, f>E$, and condition (13) is satisfied then (1) has two equilibrium points $\bar{x}_{1}=x_{-}$which is nonhyperbolic and $\bar{x}_{2}$ which is locally asymptotically stable.

(b) If $(A-e)^{2}>3 a(f-E), A>e, f>E$, and condition (14) is satisfied then the equilibrium point $\bar{x}_{1}$ is locally asymptotically stable and the equilibrium point $\bar{x}_{2}=x_{+}$is nonhyperbolic.

Proof. (a) In this case the critical points of $G(x)$ are given with (19) which yields

$$
\begin{aligned}
G\left(x_{-}\right)= & \frac{1}{27 a^{2}}\left(2(A-e)^{2}(e-A+\delta)\right) \\
& +\frac{1}{9 a}(E-f)(3(e-A)+2 \delta)-F,
\end{aligned}
$$

where $\delta=\sqrt{(A-e)^{2}-3 a(f-E)}$. Using condition (13) we obtain that $G\left(x_{-}\right)=0$ which means that $x_{-}$is an equilibrium point of (1). Since $G^{\prime}\left(x_{-}\right)=0$ we have for the equilibrium point $x_{-}=\bar{x}_{1}$ that

$$
p-1-q=0 .
$$

So we conclude that the equilibrium point $\bar{x}_{1}=(A-e-$ $\left.\sqrt{(A-e)^{2}-3 a(f-E)}\right) / 3 a$ is nonhyperbolic.

Using the fact that function $G$ is increasing when it passes through the point $\bar{x}_{2}$, we conclude that for the equilibrium point $\bar{x}_{2}$

$$
p-1-q<0 .
$$

Denote the zeros of the function $-a x^{2}+(2 A+2 e) x+E+f$ as $m_{1,2}$ :

$$
m_{1,2}=\frac{1}{a}\left(A+e \pm \sqrt{(A+e)^{2}+a(E+f)}\right) .
$$

It is clear that $m_{1}<0, m_{2}>0$. Then $p+1+q>0$ is equivalent to $\bar{x}_{2}<m_{2}$ which is equivalent to $G\left(m_{2}\right)>0$.

By using (13), one can see that

$$
\begin{aligned}
& G\left(m_{2}\right)=\frac{1}{a^{2}}\left(2 A^{3}+6 e^{3}+a e(3 E+5 f)\right. \\
& +\left(6 e^{2}+2 A^{2}+8 A e+2 a f\right) \sqrt{M} \\
& \left.+A e(10 A+14 e)+a A(E+3 f)-a^{2} F\right) \\
& \quad=\frac{1}{a^{2}}\left(2 A^{3}+6 e^{3}+a e(3 E+5 f)+6 e^{2} \sqrt{M}\right. \\
& +2 A^{2}(5 e+\sqrt{M}) \\
& \quad+A\left(14 e^{2}+a(E+3 f)+8 e \sqrt{M}\right)+2 a f \sqrt{M}
\end{aligned}
$$

$$
\begin{aligned}
& -\frac{2}{27}(A-e)^{2}(e-A+\delta)-\frac{1}{3} a(E-f)(e-A) \\
& \left.-\frac{2}{9} a \delta(E-f)\right)=\frac{1}{a^{2}}\left(2 A^{3}+6 e^{3}+\frac{8}{3} a e E+5 a e f\right. \\
& +6 e^{2} \sqrt{M}+2 A^{2}(5 e+\sqrt{M})+14 A e^{2}+a A E \\
& +\frac{8}{3} a A f+8 A e \sqrt{M}+2 a f \sqrt{M} \\
& +\frac{2}{27}(A-e)^{2}(A-e-\delta)+\frac{1}{3} a A E+\frac{1}{3} a f e \\
& \left.+\frac{2}{9} a \delta(f-E)\right)>0
\end{aligned}
$$

where $M=(A+e)^{2}+a(E+f)$. Therefore $\bar{x}_{2}$ is locally asymptotically stable.

(b) It is clear that $m_{2}>x_{+}$. Since $\bar{x}_{1}<x_{+}<m_{2}$ we conclude that for the equilibrium point $\bar{x}_{1}$

$$
\begin{aligned}
& p+1+q>0, \\
& p-1-q<0,
\end{aligned}
$$

which implies that $\bar{x}_{1}$ is locally asymptotically stable.

We have

$$
\begin{aligned}
G\left(x_{+}\right)= & -\frac{2}{27 a^{2}}(A-e)^{2}(A-e+\delta) \\
& -\frac{1}{9 a}(E-f)[3(A-e)+2 \delta]-F .
\end{aligned}
$$

Using (14) we get that $G\left(x_{+}\right)=0$ which means that $x_{+}=\bar{x}_{2}$ is an equilibrium point of (1).

Since $G^{\prime}\left(x_{+}\right)=0$ we have for the equilibrium point $\bar{x}_{2}$ that

$$
p-1-q=0,
$$

which implies that $\bar{x}_{2}$ is a nonhyperbolic equilibrium point.

Theorem 10. Assume that $F>0$ and let $M=(A+e)^{2}+a(E+$ f).

$$
\begin{aligned}
& \text { If }(A-e)^{2}>3 a(f-E), A>e, f>E, \text { and } \\
& \begin{array}{l}
27 a^{2} F^{2}-(E-f)^{2}(e-A)^{2}-4 a(E-f)^{3} \\
\quad+4 F(A-e)^{3}+18 a F(E-f)(A-e)<0
\end{array}
\end{aligned}
$$

then (1) has three equilibrium points: $\bar{x}_{1}$ which is locally asymptotically stable, $\bar{x}_{2}$ which is saddle point, and $\bar{x}_{3}$ which is

(i) locally asymptotically stable if

$$
\begin{aligned}
2 A^{3}+ & 6 e^{3}+a e(3 E+5 f) \\
& +\left(6 e^{2}+2 A^{2}+8 A e+2 a f\right) \sqrt{M}+10 A^{2} e \\
& +14 A e^{2}+a A(E+3 f)-a^{2} F>0,
\end{aligned}
$$


(ii) nonhyperbolic if

$$
\begin{aligned}
2 A^{3}+ & 6 e^{3}+a e(3 E+5 f) \\
& +\left(6 e^{2}+2 A^{2}+8 A e+2 a f\right) \sqrt{M}+10 A^{2} e \\
& +14 A e^{2}+a A(E+3 f)-a^{2} F=0,
\end{aligned}
$$

(iii) a saddle point if

$$
\begin{aligned}
2 A^{3}+ & 6 e^{3}+a e(3 E+5 f) \\
& +\left(6 e^{2}+2 A^{2}+8 A e+2 a f\right) \sqrt{M}+10 A^{2} e \\
& +14 A e^{2}+a A(E+3 f)-a^{2} F<0 .
\end{aligned}
$$

Proof. Notice that $\bar{x}_{1}<x_{-}<\bar{x}_{2}<x_{+}<\bar{x}_{3}, x_{+}<m_{2}$, where $x_{-}, x_{+}$, and $m_{2}$ are given by (19) and (33). Based on the properties of the cubic function we conclude that $G(x)$ is increasing as it passes through the point $\bar{x}_{1}$ which means that for the equilibrium point $\bar{x}_{1}$

$$
p-1-q<0 .
$$

Furthermore $G(x)$ is decreasing as it passes through the point $\bar{x}_{2}$ which implies that for the equilibrium point $\bar{x}_{2}$

$$
p-1-q>0 .
$$

The function $G(x)$ is increasing as it passes through the point $\bar{x}_{3}$, which implies that for the equilibrium point $\bar{x}_{3}$

$$
p-1-q<0 \text {. }
$$

Since $\bar{x}_{1}<\bar{x}_{2}<m_{2}$ we have that for the equilibrium points $\bar{x}_{1}$ and $\bar{x}_{2}$

$$
p+1+q>0 \text {. }
$$

So we prove that $\bar{x}_{1}$ is locally asymptotically stable equilibrium point and $\bar{x}_{2}$ is a saddle point.

Notice that for the equilibrium point $\bar{x}_{3}$

$$
p+1+q>0
$$

if $\bar{x}_{3}<m_{2}$ which is equivalent to $G\left(m_{2}\right)>0$,

$$
p+1+q=0
$$

if $\bar{x}_{3}=m_{2}$ which is equivalent to $G\left(m_{2}\right)=0$,

$$
p+1+q<0
$$

if $\bar{x}_{3}>m_{2}$ which is equivalent to $G\left(m_{2}\right)<0$.

Therefore the equilibrium point $\bar{x}_{3}$ is locally asymptotically stable if

$$
\begin{aligned}
2 A^{3}+ & 6 e^{3}+a e(3 E+5 f)+6 e^{2} \sqrt{M} \\
& +2 A^{2}(5 e+\sqrt{M}) \\
& +A\left(14 e^{2}+a(E+3 f)+8 e \sqrt{M}\right) \\
& +a(-a F+2 f \sqrt{M})>0,
\end{aligned}
$$

nonhyperbolic if

$$
\begin{aligned}
2 A^{3}+ & 6 e^{3}+a e(3 E+5 f)+6 e^{2} \sqrt{M} \\
& +2 A^{2}(5 e+\sqrt{M}) \\
& +A\left(14 e^{2}+a(E+3 f)+8 e \sqrt{M}\right) \\
& +a(-a F+2 f \sqrt{M})=0,
\end{aligned}
$$

and a saddle point if

$$
\begin{aligned}
2 A^{3}+ & 6 e^{3}+a e(3 E+5 f)+6 e^{2} \sqrt{M} \\
& +2 A^{2}(5 e+\sqrt{M}) \\
& +A\left(14 e^{2}+a(E+3 f)+8 e \sqrt{M}\right) \\
& +a(-a F+2 f \sqrt{M})<0 .
\end{aligned}
$$

\section{Case $F=0$}

This section gives complete local stability analysis for all equilibrium points (up to three) of (1) when $F=0$. In this case (1) has always the zero equilibrium.

3.1. Equilibrium Points. Equilibrium points of (1) are the solutions of the equation

$$
\bar{x}=\frac{A \bar{x}^{2}+E \bar{x}}{a \bar{x}^{2}+e \bar{x}+f}
$$

or equivalently

$$
a \bar{x}^{3}+(e-A) \bar{x}^{2}+(f-E) \bar{x}=0,
$$

which means that the equilibrium points are $\bar{x}_{0}=0$ and $\bar{x}_{ \pm}=$ $\left(A-e \pm \sqrt{(e-A)^{2}-4 a(f-E)}\right) / 2 a$.

This implies the following:

(a) If $(A-e)^{2}<4 a(f-E)$ or $A \leq e$ and $f=E$ or $(e-$ $A)^{2}=4 a(f-E)$ and $A \leq e$, there exists the unique equilibrium point $\bar{x}_{0}=0$.

(b) If $(A-e)^{2}=4 a(f-E)$ and $A>e$, there exist $\bar{x}_{0}=0$ and the positive equilibrium point $\bar{x}=(A-e) / 2 a$.

(c) If $f=E$ and $A>e$, there exist $\bar{x}_{0}=0$ and the positive equilibrium point $\bar{x}=(A-e) / a$.

(d) If $f<E$, there exist $\bar{x}_{0}=0$ and the positive equilibrium point $\bar{x}_{+}=\left(A-e+\sqrt{(e-A)^{2}-4 a(f-E)}\right) / 2 a$.

(e) If $(A-e)^{2}>4 a(f-E)$ and $f>E$ and $A>e$, there exist $\bar{x}_{0}=0$ and two positive equilibrium points $\bar{x}_{ \pm}=$ $\left(A-e \pm \sqrt{(e-A)^{2}-4 a(f-E)}\right) / 2 a$.

See Table 2 for all possible cases of existence of equilibrium points. 
TABLE 2: Existence of the equilibrium points of (1) when $F=0$.

\begin{tabular}{|c|c|c|c|}
\hline $\begin{array}{l}(A-e)^{2}<4 a(f-E) \\
(A-e)^{2}=4 a(f-E) \\
(A-e)^{2}>4 a(f-E)\end{array}$ & $\begin{array}{c}A \leq e \\
A<e, f \geq E \\
A=e, f \geq E\end{array}$ & $\begin{array}{c}\bar{x}=0 \\
\bar{x}_{ \pm}=\bar{x}=0 \\
\bar{x}_{+}=\bar{x}_{1}=0\left(\bar{x}_{-}<0\right) \text { or } \bar{x}_{ \pm}<0, \bar{x}_{1}=0 \\
\bar{x}_{1}=0\end{array}$ & One equilibrium point \\
\hline $\begin{array}{l}(A-e)^{2}>4 a(f-E) \\
(A-e)^{2}>4 a(f-E) \\
(A-e)^{2}=4 a(f-E)\end{array}$ & $\begin{array}{c}A>e, f \leq E \\
A<e, f<E \\
\quad A>e \\
A=e, f<E\end{array}$ & $\begin{array}{c}\bar{x}_{1}=\bar{x}_{-}=0, \bar{x}_{+}>0 \text { or } \bar{x}_{-}<0, \bar{x}_{1}=0, \bar{x}_{+}>0 \\
\bar{x}_{-}<0, \bar{x}_{1}=0, \bar{x}_{+}>0 \\
\bar{x}_{+}=\bar{x}_{-}>0, \bar{x}_{1}=0 \\
\bar{x}_{1}=0, \bar{x}_{+}>0\end{array}$ & Two equilibrium points \\
\hline$(A-e)^{2}>4 a(f-E)$ & $A>e, f>E$ & $\bar{x}_{1}=0,0<\bar{x}_{-}<\bar{x}_{+}$ & Three equilibrium points \\
\hline
\end{tabular}

Remark 11. It should be noticed that conditions on existence of one, two, or three equilibrium points for (1) summarized in Tables 1 and 2 can be extended to the corresponding results for the general quadratic fractional difference equation (3). Indeed, the equilibrium points of (3) satisfy the equilibrium equation

$$
\begin{gathered}
(a+b+c) x^{3}+(d+e-A-B-C) x^{2} \\
+(f-D-E) x-F=0
\end{gathered}
$$

Thus replacing the coefficients $a$ with $a+b+c, e$ with $d+e$, $A$ with $A+B+C$, and $E$ with $D+E$ in Tables 1 and 2, we can obtain the explicit conditions for the existence of one, two, or three equilibrium points. The complement of the union of such conditions will give the condition for nonexistence of equilibrium points.

3.2. Local Stability Analysis. The linearized equation of (1) at the zero equilibrium is of the form

$$
z_{n+1}=p z_{n}+q z_{n-1}
$$

where $p=(\partial H / \partial u)(0,0)=0$ and $q=(\partial H / \partial v)(0,0)=E / f$, if we denote $f(u, v)=\left(A u^{2}+E v\right) /\left(a u^{2}+e v+f\right)$.

Now we have the following result on local stability of the zero equilibrium (see Theorem 1.1 from [1]).

Proposition 12. The zero equilibrium of (1) is one of the following:

(a) locally asymptotically stable if $E<f$,

(b) nonhyperbolic of resonance type $(1,-1)$ if $E=f$,

(c) repeller if $E>f$.

The linearized equation of (1) at the positive equilibrium point is of the form

$$
z_{n+1}=\frac{2 \bar{x}(A-a \bar{x})}{A \bar{x}+E} z_{n}+\frac{E-e \bar{x}}{A \bar{x}+E} z_{n-1}
$$

Indeed, since

$$
a \bar{x}^{2}+e \bar{x}+f=A \bar{x}+E
$$

we have that

$$
\begin{aligned}
p & =H_{u}^{\prime}(\bar{x}, \bar{x}) \\
& =\frac{2 A \bar{x}\left(a \bar{x}^{2}+e \bar{x}+f\right)-2 a \bar{x}\left(A \bar{x}^{2}+E \bar{x}\right)}{\left(a \bar{x}^{2}+e \bar{x}+f\right)^{2}} \\
& =\frac{2 \bar{x}(A-a \bar{x})}{A \bar{x}+E}, \\
q & =H_{v}^{\prime}(\bar{x}, \bar{x})=\frac{E\left(a \bar{x}^{2}+e \bar{x}+f\right)-e \bar{x}(A \bar{x}+E)}{\left(a \bar{x}^{2}+e \bar{x}+f\right)^{2}} \\
& =\frac{E-e \bar{x}}{A \bar{x}+E} .
\end{aligned}
$$

Now we have that

$$
\begin{aligned}
p+q-1 & =\frac{2 \bar{x}(A-a \bar{x})}{A \bar{x}+E}+\frac{E-e \bar{x}}{A \bar{x}+E}-1 \\
& =\frac{\bar{x}(A-2 a \bar{x}-e)}{A \bar{x}+E}=\frac{-a \bar{x}^{2}+f-E}{A \bar{x}+E}, \\
p-q+1 & =\frac{3 A \bar{x}+e \bar{x}-2 a \bar{x}^{2}}{A \bar{x}+E}=\frac{\bar{x}(3 A-2 a \bar{x}+e)}{A \bar{x}+E}, \\
q-1 & =\frac{E-e \bar{x}}{A \bar{x}+E}-1=-\frac{\bar{x}(A+e)}{A \bar{x}+E}<0, \\
q+1 & =\frac{E-e \bar{x}}{A \bar{x}+E}+1=\frac{(A-e) \bar{x}+2 E}{A \bar{x}+E},
\end{aligned}
$$

which shows that $q<1$.

Theorem 13. (a) If $A=e$ and $E>f$, then (1) has two equilibrium points: $\bar{x}_{0}=0$ which is repeller and $\bar{x}^{*}=$ $\sqrt{(E-f) / a}$ which is locally asymptotically stable.

(b) If $(e-A)^{2}=4 a(f-E)$ and $A>e$, then (1) has two equilibrium points: $\bar{x}_{0}$ which is locally asymptotically stable and $\bar{x}_{+}$which is nonhyperbolic and locally stable.

(c) If $A>e$ and $f=E$, then (1) has two equilibrium points: $\bar{x}_{0}$ which is nonhyperbolic and locally stable and $\bar{x}_{*}=(A-e) / a$ which is locally asymptotically stable. 
Proof. (a) In view of Proposition 12 we have that $\bar{x}_{0}$ is repeller. It follows from (67) and (70) that for the equilibrium point $\bar{x}^{*}$

$$
\begin{aligned}
p+q-1 & =\frac{\bar{x}(A-e-2 a \bar{x})}{A \bar{x}+E}=-\frac{2 a \bar{x}^{2}}{A \bar{x}+E}<0, \\
q+1 & =\frac{a \bar{x}^{2}+f-A \bar{x}}{a \bar{x}^{2}+e \bar{x}+f}+1 \\
& =\frac{2 a \bar{x}^{2}+(e-A) \bar{x}+2 f}{a \bar{x}^{2}+e \bar{x}+f}=\frac{2 a \bar{x}^{2}+2 f}{a \bar{x}^{2}+e \bar{x}+f} \\
& >0,
\end{aligned}
$$

which shows that $\bar{x}^{*}$ is locally asymptotically stable.

(b) By assumption we have that $E<f$. Because of that $\bar{x}_{0}$ is locally asymptotically stable (see Proposition $12(\mathrm{a})$ ).

For the equilibrium point $\bar{x}_{+}=(A-e) / 2 a$ we obtain from (67) and (70) that

$$
\begin{aligned}
p+q-1 & =\frac{(A-e) \bar{x}-2 a \bar{x}^{2}}{A \bar{x}+E} \\
& =\frac{(A-e) \bar{x}-2 a \bar{x}((A-e) / 2 a)}{A \bar{x}+E}=0
\end{aligned}
$$

and $q>-1$.

The proof follows from Theorem 1.1 [1].

(c) Since $f=E$ the zero equilibrium is nonhyperbolic and locally stable (see Proposition 12(b)). For the equilibrium point $\bar{x}_{*}=(A-e) / a$ we have that

$$
p+q-1=\frac{\bar{x}(e-A)}{A \bar{x}+E}=-\frac{a \bar{x}^{2}}{A \bar{x}+E}<0
$$

which by (72) implies $q+1>0$. This means that the equilibrium point $\bar{x}_{*}$ is locally asymptotically stable.

Theorem 14. Assume that $f<E$. Then (1) has two equilibrium points $\bar{x}_{0}$ which is repeller and $\bar{x}_{+}$which is one of the following:

(i) If $3 A^{2}+3 e^{2}+6 A e>4 a(E-f)$, then $\bar{x}_{+}$is locally asymptotically stable.

(ii) If $3 A^{2}+3 e^{2}+6 A e=4 a(E-f)$, then $\bar{x}_{+}$is nonhyperbolic.

(iii) If $3 A^{2}+3 e^{2}+6 A e<4 a(E-f)$, then $\bar{x}_{+}$is saddle point.
Proof. Proposition 12 implies that $\bar{x}_{0}$ is a repeller. Note that for $\bar{x}=\bar{x}_{+} p$ can take values of distinct signs. We have

$$
\begin{aligned}
p+q-1 & =\frac{\bar{x}(A-e-2 a \bar{x})}{A \bar{x}+E} \\
& =\frac{\bar{x}(A-e-A+e-\sqrt{\Delta})}{A \bar{x}+E}=-\frac{\sqrt{\Delta} \bar{x}}{A \bar{x}+E} \\
& <0, \\
p-q+1 & =\frac{\bar{x}(3 A+e-2 a \bar{x})}{A \bar{x}+E}=\frac{\bar{x}(2 A+2 e-\sqrt{\Delta})}{A \bar{x}+E}, \\
q+1 & =\frac{2 a \bar{x}^{2}+(e-A) \bar{x}+2 f}{a \bar{x}^{2}+e \bar{x}+f} \\
& =\frac{(A-e+\sqrt{\Delta}) \bar{x}+(e-A) \bar{x}+2 f}{a \bar{x}^{2}+e \bar{x}+f} \\
& =\frac{\bar{x} \sqrt{\Delta}+2 f}{a \bar{x}^{2}+e \bar{x}+f}>0 .
\end{aligned}
$$

Since

$$
\begin{aligned}
2 A+2 e-\sqrt{\Delta} & >0 \Longleftrightarrow \\
4(A+e)^{2} & >(A-e)^{2}+4 a(E-f) \Longleftrightarrow \\
3 A^{2}+3 e^{2}+6 A e & >4 a(A-f)
\end{aligned}
$$

the proof follows from Theorem 1.1 [1].

Theorem 15. Assume that $(e-A)^{2}>4 a(f-E), f>E$, and $A>e$. Then (1) has three equilibrium points: $\bar{x}_{0}$ which is locally asymptotically stable, $\bar{x}_{-}$which is a saddle point, and $\bar{x}_{+}$which is locally asymptotically stable.

Proof. For the equilibrium point $\bar{x}_{+}$we have

$$
\begin{aligned}
p+q-1 & =-\frac{\sqrt{\Delta} \bar{x}}{A \bar{x}+E}<0, \\
q+1 & =\frac{\bar{x} \sqrt{\Delta}+2 f}{a \bar{x}^{2}+e \bar{x}+f}>0, \\
p-q+1 & =\frac{\bar{x}(2 A+2 e-\sqrt{\Delta})}{A \bar{x}+E}>0,
\end{aligned}
$$

because

$$
2 A+2 e>\sqrt{\Delta} \Longleftrightarrow
$$$$
3 A^{2}+3 e^{2}+6 A e+4 a(f-E)>0
$$

which is satisfied under assumptions of theorem. It means that $\bar{x}_{+}$is locally asymptotically stable. 
For the equilibrium point $\bar{x}_{-}$we have

$$
\begin{aligned}
& p+q-1=\frac{\bar{x} \sqrt{\Delta}}{A \bar{x}+E}>0, \\
& p-q+1=\frac{\bar{x}(2 A+2 e+\sqrt{\Delta})}{A \bar{x}+E}>0,
\end{aligned}
$$

which implies that $\bar{x}_{-}$is saddle point.

The equilibrium point $\bar{x}_{0}$ is locally asymptotically stable by Proposition 12.

\section{Global Asymptotic Stability Results}

In this section we give some global asymptotic stability results for some special cases of (1).

Theorem 16. Consider (1), where all coefficients are positive, subject to the condition

$$
\frac{|A-a \bar{x}|(U+\bar{x})+|E-e \bar{x}|}{a L^{2}+e L+f}<1,
$$

where $L=\min \{A, E, F\} / \max \{a, e, f\}$ and $U=$ $\max \{A, E, F\} / \min \{a, e, f\}$, and assume the hypotheses of Theorem $5(a)$. Then $\bar{x}$ is globally asymptotically stable.

Proof. In view of Corollary 3 we need to find the lower and upper bounds for all solutions of (1) for $n \geq 1$. In this case the lower and upper bounds for all solutions of (1) for $n \geq 1$ are derived as

$$
\begin{aligned}
x_{n+1} & =\frac{A x_{n}^{2}+E x_{n-1}+F}{a x_{n}^{2}+e x_{n-1}+f} \\
& \leq \frac{\max \{A, E, F\}\left(x_{n}^{2}+x_{n-1}+1\right)}{\min \{a, e, f\}\left(x_{n}^{2}+x_{n-1}+1\right)} \\
& =\frac{\max \{A, E, F\}}{\min \{a, e, f\}}=U, \\
x_{n+1} & =\frac{A x_{n}^{2}+E x_{n-1}+F}{a x_{n}^{2}+e x_{n-1}+f} \\
& \geq \frac{\min \{A, E, F\}\left(x_{n}^{2}+x_{n-1}+1\right)}{\max \{a, e, f\}\left(x_{n}^{2}+x_{n-1}+1\right)} \\
& =\frac{\min \{A, E, F\}}{\max \{a, e, f\}}=L .
\end{aligned}
$$

Theorem 17. Consider (1), where $E=0$ and all other coefficients are positive, subject to the condition

$$
\frac{|A-a \bar{x}|(U+\bar{x})+e \bar{x}}{a L^{2}+e L+f}<1,
$$

where $L=0$ and $U=A / a+F / f$ and

(i) $(A-e)^{2}>3$ af and $A<e$; (ii) $(A-e)^{2}>3 a f, A>e$, and $4 a f^{3}-f^{2}(e-A)^{2}+4 F(A-$ $e)^{3}-18 a f F(A-e)+27 a^{2} F^{2}>0$;

(iii) $(A-e)^{2} \leq 3 a f$.

Then $\bar{x}$ is globally asymptotically stable.

Proof. In this case, lower and upper bounds of all solutions of (1) are given with

$$
\begin{aligned}
L & =0 \leq x_{n+1}=\frac{A x_{n}^{2}+F}{a x_{n}^{2}+e x_{n-1}+f} \\
& =\frac{A x_{n}^{2}}{a x_{n}^{2}+e x_{n-1}+f}+\frac{F}{a x_{n}^{2}+e x_{n-1}+f} \leq \frac{A}{a}+\frac{F}{f}
\end{aligned}
$$$$
=U \text {. }
$$

Now, an application of Theorem 5 and Corollary 3 completes the proof.

Theorem 18. Consider (1), where $A=0$ and all other coefficients are positive, subject to the condition

$$
a \bar{x}(U+\bar{x})+|E-e \bar{x}|<f
$$

where $L=0$ and $U=\max \{E, F\} / \min \{e, f\}$. Then $\bar{x}$ is globally asymptotically stable.

Proof. In this case, in view of Theorem 5(a) part (1), (1) has the unique equilibrium. Lower and upper bounds of all solutions of (1) are given with

$$
\begin{aligned}
L & =0<x_{n+1}=\frac{E x_{n-1}+F}{a x_{n}^{2}+e x_{n-1}+f} \leq \frac{E x_{n-1}+F}{e x_{n-1}+f} \\
& \leq \frac{\max \{E, F\}\left(x_{n-1}+1\right)}{\min \{e, f\}\left(x_{n-1}+1\right)}=\frac{\max \{E, F\}}{\min \{e, f\}}=U .
\end{aligned}
$$

Now, an application of Theorem 5 part (3) and Corollary 3 completes the proof.

Theorem 19. Consider (1), where $f=0$ and all other coefficients are positive, subject to the condition

$$
\frac{|A-a \bar{x}|(U+\bar{x})+|E-e \bar{x}|}{a L^{2}+e L}<1,
$$

where $L=\min \{A, E\} / \max \{a, e\}$ and $U=\max \{A, E\} /$ $\min \{a, e\}+F /\left(a L^{2}+e L\right)$. Then $\bar{x}$ is globally asymptotically stable. 
Proof. In this case, in view of Theorem 5(a) part (1), (1) has the unique equilibrium. Lower and upper bounds of all solutions of (1) are given with

$$
\begin{aligned}
x_{n+1} & =\frac{A x_{n}^{2}+E x_{n-1}+F}{a x_{n}^{2}+e x_{n-1}} \geq \frac{A x_{n}^{2}+E x_{n-1}}{a x_{n}^{2}+e x_{n-1}} \\
& \geq \frac{\min \{A, E\}\left(x_{n}^{2}+x_{n-1}\right)}{\max \{a, e\}\left(x_{n}^{2}+x_{n-1}\right)}=\frac{\min \{A, E\}}{\max \{a, e\}}=L, \\
x_{n+1} & =\frac{A x_{n}^{2}+E x_{n-1}+F}{a x_{n}^{2}+e x_{n-1}} \\
& =\frac{A x_{n}^{2}+E x_{n-1}}{a x_{n}^{2}+e x_{n-1}}+\frac{F}{a x_{n}^{2}+e x_{n-1}} \\
& \leq \frac{\max \{A, E\}}{\min \{a, e\}}+\frac{F}{a L^{2}+e L}=U .
\end{aligned}
$$

Now, an application of Corollary 3 completes the proof.

Theorem 20. Consider (1), where $F=0$ and all other coefficients are positive, subject to the condition

$$
|A-a \bar{x}|(U+\bar{x})+|E-e \bar{x}|<f,
$$

where $L=0, U=\max \{A, E\} / \min \{a, e\}$, and

(i) $(A-e)^{2}>4 a(f-E)$ and $A<e, f \geq E$,

(ii) $(A-e)^{2}=4 a(f-E)$ and $A \leq e$,

(iii) $(A-e)^{2}<4 a(f-E)$,

(iv) $A=e, E \leq f$.

Then $\bar{x}=0$ is globally asymptotically stable.

Proof. In these cases (1) has the unique equilibrium point $\bar{x}=$ 0 and lower and upper bounds of all solutions of (1) are given with

$$
\begin{aligned}
L & =0 \leq x_{n+1}=\frac{A x_{n}^{2}+E x_{n-1}}{a x_{n}^{2}+e x_{n-1}+f}<\frac{A x_{n}^{2}+E x_{n-1}}{a x_{n}^{2}+e x_{n-1}} \\
& \leq \frac{\max \{A, E\}\left(x_{n}^{2}+x_{n-1}\right)}{\min \{a, e\}\left(x_{n}^{2}+x_{n-1}\right)}=\frac{\max \{A, E\}}{\min \{a, e\}}=U .
\end{aligned}
$$

Now, an application of Corollary 3 completes the proof.

Theorem 21. Consider (1), where $A=E=0$ and all other coefficients are positive, subject to the condition

$$
a \bar{x}(U+\bar{x})+e \bar{x}<f,
$$

where $L=0$ and $U=F / f$. Then $\bar{x}$ is globally asymptotically stable.

Proof. In this case (1) has the unique equilibrium. Lower and upper bounds of all solutions of (1) are given with

$$
L=0 \leq x_{n+1}=\frac{F}{a x_{n}^{2}+e x_{n-1}+f} \leq \frac{F}{f}=U,
$$

and an application of Corollary 3 completes the proof.
Simpler and more effective global asymptotic stability results (although less general) can be obtained by applying Theorems 1.4.5-1.4.8 in [1]. For instance, consider (1), where $A=E=0$ and all other coefficients are positive; that is, consider the equation

$$
\begin{aligned}
x_{n+1}=\frac{F}{x_{n}^{2}+e x_{n-1}+f}=G\left(x_{n}, x_{n-1}\right), & \\
& n=0,1,2, \ldots,
\end{aligned}
$$

where we can assume that $a=1$. Then the following result holds.

Theorem 22. Consider (92), subject to the condition

$$
\begin{array}{r}
F \leq e f \text { or } \\
(F-e f)^{2} \leq 4 f^{3} .
\end{array}
$$

Then $\bar{x}$ is globally asymptotically stable.

Proof. The function

$$
G(u, v)=\frac{F}{u^{2}+e v+f}
$$

is decreasing in both arguments and it has an invariant interval $I=[0, F / f]$ with the property that $G: I^{2} \rightarrow I$. Furthermore $I$ is also an attracting interval; that is, $x_{n} \in I$, $n \geq 1$, for every solution $\left\{x_{n}\right\}$ of (92). In order to apply Theorem 1.4.7 in [1] we need to show that the only solution $(m, M) \in I^{2}$ of the system

$$
\begin{aligned}
M & =G(m, m), \\
m & =G(M, M)
\end{aligned}
$$

is the equilibrium solution $M=m=\bar{x}$. System (95) implies

$$
M m^{2}+f M=m M^{2}+f m=F-e m M
$$

and $(m-M)(M m-f)=0$. If $M m \neq f$, then $M=m$. If $M m=f$, then system (95) gives $f(m+M)=F-e f$, which is impossible if $F \leq e f$. However, if $F>e f$, then $m$ and $M$ are solutions of the quadratic equation

$$
x^{2}-\frac{F-e f}{f} x+f=0,
$$

obtained by elimination of $M$ from system (95). Equation $x^{2}-((F-e f) / f) x+f=0$ has no two real solutions if its discriminant $(F-e f)^{2}-4 f^{3}$ is nonpositive.

Another special case of (1) which exhibits the global asymptotic stability of the unique equilibrium is the following equation:

$$
x_{n+1}=\frac{x_{n}^{2}}{x_{n}^{2}+e x_{n-1}+f}=H\left(x_{n}, x_{n-1}\right),
$$

$$
n=0,1,2, \ldots,
$$

where we can assume that $a=A=1$. 
Theorem 23. Consider (98), subject to the condition

$$
e \geq 1
$$

Then $\bar{x}=0$ is globally asymptotically stable.

Proof. The function

$$
H(u, v)=\frac{u^{2}}{u^{2}+e v+f}
$$

is increasing in $u$ and decreasing in $v$ and it has an invariant interval $I=[0,1]$ with the property that $H: I^{2} \rightarrow I$. Furthermore $I$ is also an attracting interval; that is, $x_{n} \in I$, $n \geq 1$, for every solution $\left\{x_{n}\right\}$ of (98). Equation (98) has only the zero equilibrium under the condition (99). Indeed the equilibrium solutions of (98) satisfy $x\left(x^{2}+(e-1) x+f\right)=0$, and so there is no positive equilibrium if $e \geq 1$. In order to apply Theorem 1.4.5 in [1] we need to show that the only solution $(m, M) \in I^{2}$ of the system

$$
\begin{gathered}
M=H(M, m), \\
m=H(m, M)
\end{gathered}
$$

is the equilibrium solution $M=m=0$. System (95) implies

$$
\begin{aligned}
& e m=M-M^{2}-f \\
& e M=m-m^{2}-f
\end{aligned}
$$

and $(m-M)(e+1-M-m)=0$. If $M+m \neq e+1$, then $M=m$. If $M+m=e+1$, then system (101) implies that $m$ and $M$ are solutions of the quadratic equation

$$
x^{2}-(e+1) x+f+e(e+1)=0,
$$

obtained by elimination of $M$ from system (101). Equation $x^{2}-(e+1) x+f+e(e+1)=0$ has no two real solutions if its discriminant $(e+1)(1-3 e)-4 f$ is nonpositive, which is true if (99) holds.

\section{Conflict of Interests}

The authors declare that there is no conflict of interests regarding the publication of this paper.

\section{References}

[1] M. R. S. Kulenović and G. Ladas, Dynamics of Second Order Rational Difference Equations with Open Problems and Conjectures, Chapman \& Hall/CRC, Boca Raton, Fla, USA, 2001.

[2] S. N. Elaydi, An Introduction to Difference Equations, Undergraduate Texts in Mathematics, Springer, New York, NY, USA, 3rd edition, 2005.

[3] S. N. Elaydi, Discrete Chaos. With Applications in Science and Engineering, Chapman \& Hall/CRC, Boca Raton, Fla, USA, 2nd edition, 2008.

[4] J. Bektešević, M. R. S. Kulenović, and E. Pilav, "Global dynamics of quadratic second order difference equation in the first quadrant," Applied Mathematics and Computation, vol. 227, pp. 50-65, 2014.
[5] A. M. Amleh, E. Camouzis, and G. Ladas, "On the dynamics of a rational difference equation, part I," International Journal of Difference Equations, vol. 3, no. 1, pp. 1-35, 2008.

[6] A. M. Amleh, E. Camouzis, and G. Ladas, "On the dynamics of a rational difference equation, part II," International Journal of Difference Equations, vol. 3, no. 2, pp. 195-225, 2008.

[7] D. M. Chan, C. M. Kent, and N. L. Ortiz-Robinson, "Convergence results on a second-order rational difference equation with quadratic terms," Advances in Difference Equations, vol. 2009, Article ID 985161, 7 pages, 2009.

[8] M. Dehghan, C. M. Kent, R. Mazrooei-Sebdani, N. L. Ortiz, and H. Sedaghat, "Monotone and oscillatory solutions of a rational difference equation containing quadratic terms," Journal of Difference Equations and Applications, vol. 14, no. 10-11, pp. 1045-1058, 2008.

[9] M. Dehghan, R. Mazrooei-Sebdani, and H. Sedaghat, "Global behaviour of the Riccati difference equation of order two," Journal of Difference Equations and Applications, vol. 17, no. 4, pp. 467-477, 2011.

[10] S. Kalabušić, M. R. Kulenović, and M. Mehuljić, "Global perioddoubling bifurcation of quadratic fractional second order difference equation," Discrete Dynamics in Nature and Society, vol. 2014, Article ID 920410, 13 pages, 2014.

[11] C. M. Kent and H. Sedaghat, "Global attractivity in a quadraticlinear rational difference equation with delay," Journal of Difference Equations and Applications, vol. 15, no. 10, pp. 913-925, 2009.

[12] C. M. Kent and H. Sedaghat, "Global attractivity in a rational delay difference equation with quadratic terms," Journal of Difference Equations and Applications, vol. 17, no. 4, pp. 457-466, 2011.

[13] M. R. S. Kulenović, E. Pilav, and E. Silić, "Local dynamics and global attractivity of a certain second-order quadratic fractional difference equation," Advances in Difference Equations, vol. 2014, article 68, 32 pages, 2014.

[14] R. Mazrooei-Sebdani, "Convergence in homogeneous difference equations of degree 1," Journal of Difference Equations and Applications, vol. 19, no. 1, pp. 13-26, 2013.

[15] R. Mazrooei-Sebdani, "Homogeneous rational systems of difference equations in the plane," Journal of Difference Equations and Applications, vol. 19, no. 2, pp. 273-303, 2013.

[16] H. Sedaghat, "Global behaviours of rational difference equations of orders two and three with quadratic terms," Journal of Difference Equations and Applications, vol. 15, no. 3, pp. 215-224, 2009.

[17] H. Sedaghat, Nonlinear Difference Equations: Theory with Applications to Social Science Models, vol. 15 of Mathematical Modelling: Theory and Applications, Kluwer Academic, Dordrecht, The Netherlands, 2003.

[18] S. J. Hrustić, M. R. Kulenović, and M. Nurkanović, "Global dynamics and bifurcations of certain second order rational difference equation with quadratic terms," Qualitative Theory of Dynamical Systems, pp. 1-25, 2015.

[19] S. Jašarević Hrustić, M. R. S. Kulenović, Z. Nurkanović, and E. Pilav, "Birkhoff normal forms, KAM theory and symmetries for certain second order rational difference equation with quadratic term," International Journal of Difference Equations, vol. 10, pp. 181-199, 2015.

[20] M. DiPippo, E. J. Janowski, and M. R. S. Kulenović, "Global asymptotic stability for quadratic fractional difference equation," Advances in Difference Equations, vol. 2015, article 179, 2015. 


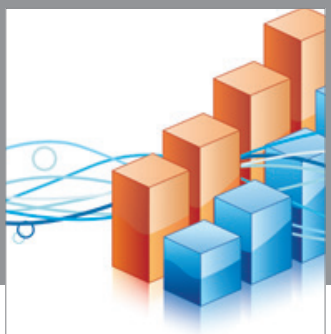

Advances in

Operations Research

vatem alat4

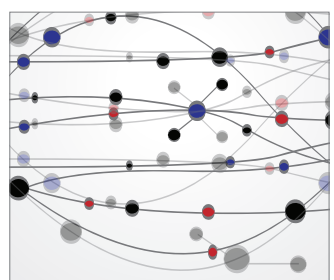

\section{The Scientific} World Journal
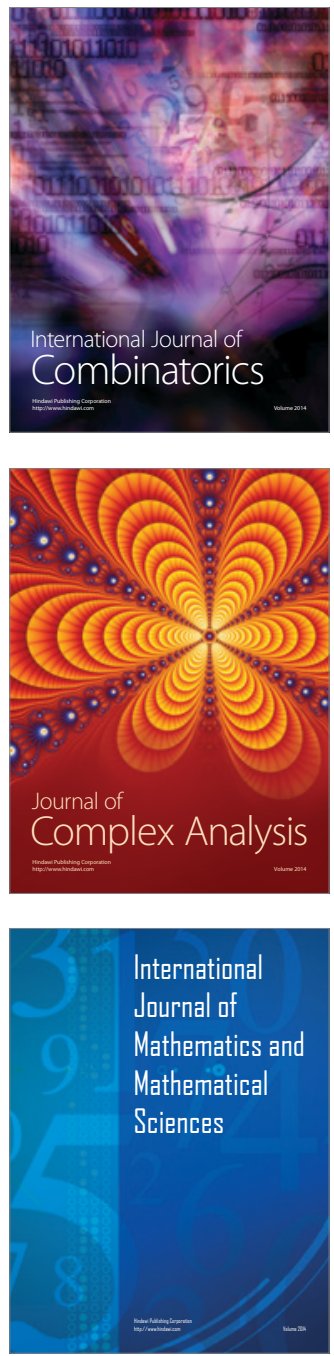
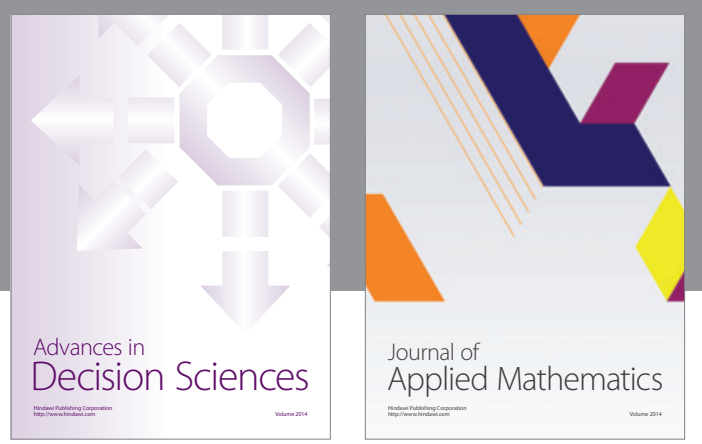

Algebra

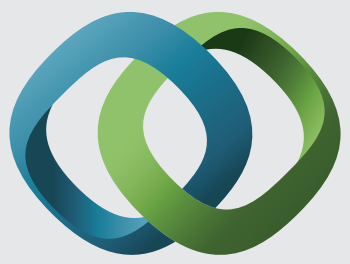

\section{Hindawi}

Submit your manuscripts at

http://www.hindawi.com
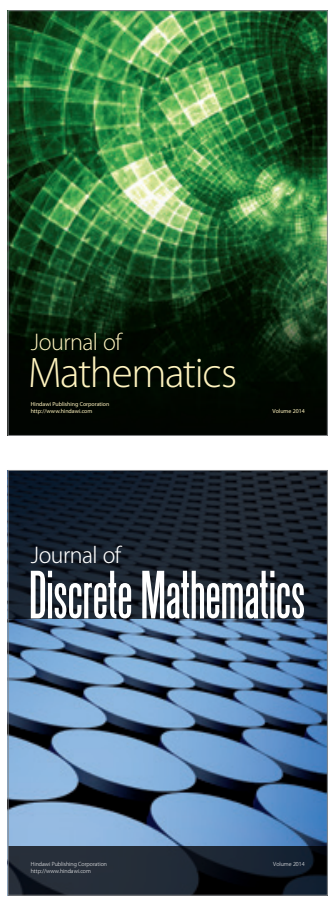

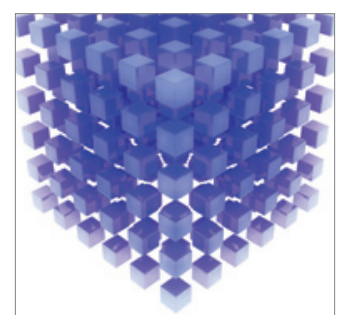

Mathematical Problems in Engineering
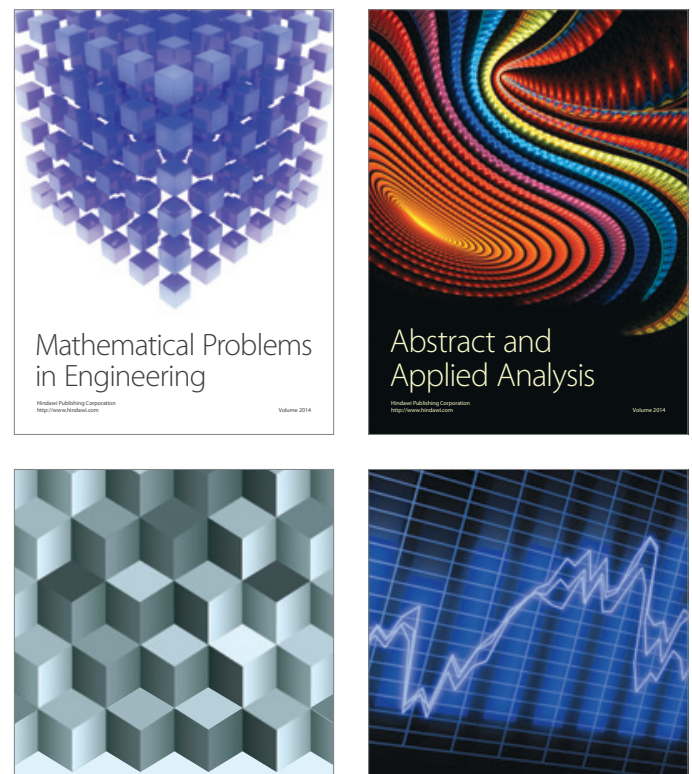

Journal of

Function Spaces

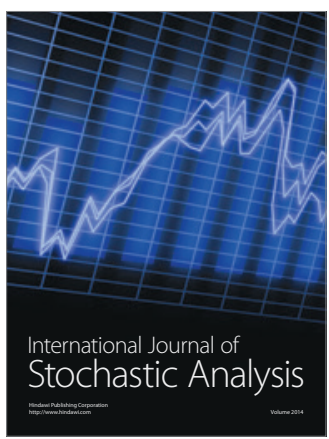

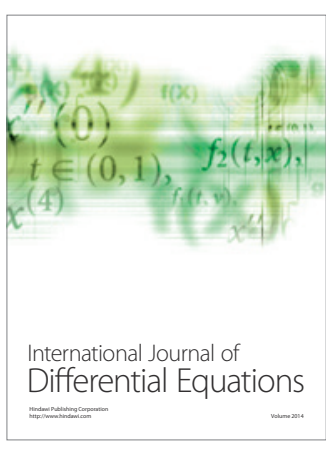
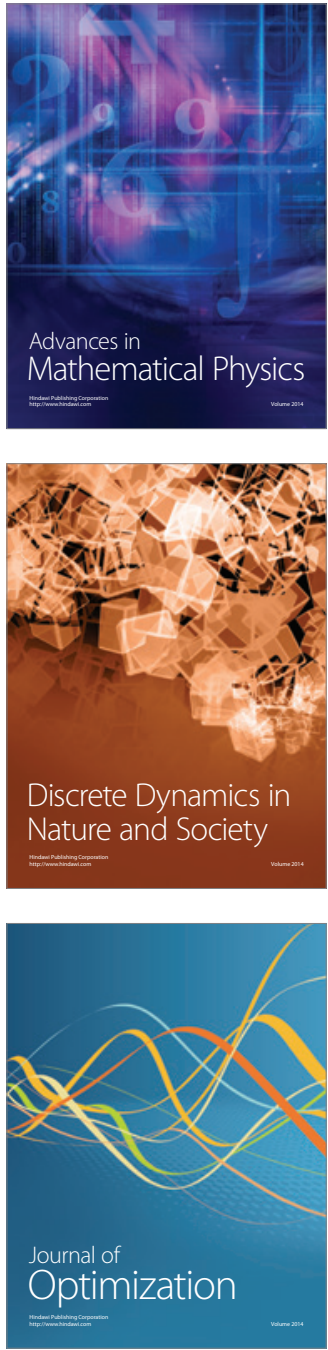